\title{
Robust Facial Data Recognition using Multimodal Fusion Features in Multi-Variant Face Acquisition
}

\author{
A.Manchula, \\ Research Scholar
}

\author{
S.Arumugam, $\mathrm{PhD}$. \\ Principal
}

\author{
Nanda College of Technology
}

\begin{abstract}
Biometric use physiological traits such as fingerprints, face and behavioral traits such as voice, hand signatures characteristics to verify an individual's identity. The two process involved in biometrics are verification and identification. Verification process is performed by matching an individual's biometric with the template of claimed identity only. The identification process performed by matching an individual's biometric with template of every identity in the database. Existing work presented a multimodal (2D and 3D) face recognition algorithm by way of performing hybrid matching which was based on both feature and holistic metrics. The Pose of 3D face and its texture corrected using single automatically detected point and hotelling transform. 3D Spherical Face Representation (SFR) is used in conjunction with Scale-Invariant Feature Transform (SIFT) descriptor which results in formation of a rejection classifier. It quickly eliminates a large number of candidate faces at an early stage for efficient recognition for known faces as attributes. Our proposed work presented a Feature Based Multimodality Face Recognition System to recognize the human individuals in environment of known faces using features like shape of the eyes, nose and jaw. Case study and preliminary experimental results conducted in Mat lab proves to be a viable approach using multimodality method based on $2 \mathrm{D}$ and $3 \mathrm{D}$ facial representation for known faces. Facial recognition rate is measured in terms of validation index generated for false acceptance rate, false rejection rate.
\end{abstract}

\section{Keywords}

Biometrics, face recognition, spherical face representation, SIFT, ICP.

\section{INTRODUCTION}

The human face is an easily collectible, universal, and non intrusive biometric feature. Fingerprinting or scanning of iris images is impractical or undesirable due to the problems of social acceptance. Face recognition is the most challenging problem due to diversity nature in faces and variations caused by several factors such as expressions, gender, pose, illumination, and makeup. Biometrics consists of methods for identification of humans based on the nature of physical or behavioral traits.

A biometric scheme works in the two ways. They are verification mode and identification mode as in the following figure 1 . The verification process is performed in order to check, such that the individual is the actual person and is conducted by matching an individual's biometric with the template of claimed identity only. The templates can be of a smart card, user name or user id which can be used for comparison. The verification process involves one-to-one comparison. The common use of verification mode is "positive recognition".

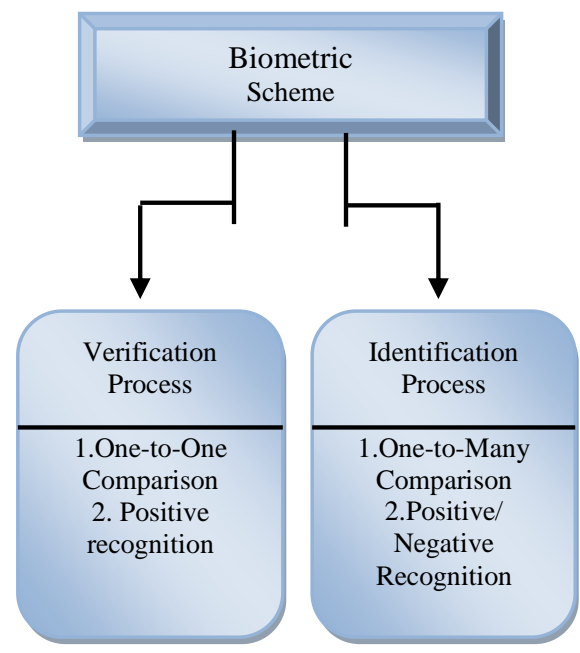

Fig 1. Conceptual Biometric scheme

In identification mode, the process involves one-to-many comparison which involves a comparison against a biometric database by matching an individual's biometric with the template of every identity in the database. Comparison results are based on the value of threshold. The process will succeed if the comparison of biometric data to a stored template lies within the threshold value. The common use of identification mode is either "positive recognition" or for "negative recognition".

\section{LITERATURE REVIEW}

Traditional face recognition techniques mainly depend on low-resolution face images, which results in the lost of important information that are contained in the microscopic traits. In paper [1], introduce a multilayer framework for high resolution face recognition system that exploits features in multiple scales. Each face image is factorized into four layers: global appearance, facial organs, skins, and irregular details. Various feature matching algorithms have been proposed for this purpose. However, the algorithms suffer from various limitations like applicability, efficiency, robustness to resolution, and the discriminating capability of the used feature representation. In [5] we present a novel feature matching algorithm for automatic pair-wise registration of range images which overcomes these limitations.

A various number of new face recognition techniques have been proposed. Some of them include recognition from three- 
dimensional (3D) scans, recognition from high resolution still images, recognition from multiple still images,

multi-modal face recognition, multi-algorithm, and preprocessing algorithms to correct for illumination and pose variations. The paper [10] discusses about Face Recognition Vendor Test (FRVT).

Current 2D face recognition systems achieve good performance in constrained environments however they face with difficulties while handling large amounts of facial variations such as head pose, lighting conditions and facial expressions. As the human face is a $3 \mathrm{D}$ object whose $2 \mathrm{D}$ projection is sensitive to the above changes, utilizing 3D facial information improves the face recognition performance. This paper [4] present with the Geometrix Face Vision3D face recognition technology, describes how shape and texture are fused. In paper [6], we present a multimodal hybrid face recognition algorithm and illustrate its performance on the FRGC v1.0 data. We use hybrid model feature-based and holistic matching for the 3D faces and a holistic matching approach on the $2 \mathrm{D}$ faces.

In paper [2] presents technique for extracting distinctive invariant features from images which can be used to perform matching between different views of an object. This approach has been called as Scale Invariant Feature Transform (SIFT). For image matching and recognition, SIFT features are first extracted from a set of reference images and stored in a database. A new image is matched by comparing each feature from the new image to this previous database and finding candidate matching features based on Euclidean distance of their feature vectors. The paper [2] discusses fast nearestneighbor algorithms that can perform this computation rapidly against large databases.

The performance of face recognition systems that use 2D images depends on factors such as lighting and subject's pose. This paper [3] develops a face recognition system that utilizes three-dimensional shape information to make the system more robust to arbitrary pose and lighting. For each step, a 3D face model is constructed by combining several $2.5 \mathrm{D}$ face scans which are captured from different views. A fully automatic 3D face recognition algorithm is presented in paper [7]. Several novelties have been included in this paper. These include automatic 3D face detection, automatic pose correction and normalization of the 3D face, a Spherical Face Representation and its use as a rejection classifier and robustness to facial expressions.

Paper [8] discusses about the multi-view correspondence algorithm which automatically establishes correspondences between the unordered $2.5 \mathrm{D}$ views of a free-form object by performing a one-to-many correspondence search using a 4D hash table. This result in a spanning tree of relative transformations between the unordered views used to coarsely register them in a common coordinate basis. The registration process is further refined using multi-view fine registration followed by the integration and reconstruction of the views into a seamless 3D model.

Multimodal approaches either combine 3D geometry with 2D texture or with 2D IR data. Most of these works employ small datasets and do not focus on handling facial expressions. The paper [9] focuses on large datasets by way of using wavelet analysis to extract a compact biometric signature, thus allowing us to perform rapid comparisons on either a global or a per area basis. Data normalization is applied separately on the $2 \mathrm{D}$ and $3 \mathrm{D}$ data. And the normalized images are used for the PCA and edge-based approaches. The ICP-based approach does not require such extensive normalization. Details of the steps for normalization can be found in [11].

\section{FEATURE BASED MULTIMODAL FACE RECOGNITION (FBMFRS)}

The work presented a multimodal (2D and 3D) face recognition algorithm and matching algorithm based on hybrid model using both feature and holistic metrics. The pose of 3D face and its texture is corrected using single automatically detected point and by using hotelling transform. Both 3D Spherical Face Representation (SFR) and Scale Invariant Feature Transform (SIFT) description are used to form a rejection classifier. SFR is compared with two existing $3 \mathrm{D}$ representations like spin and tensor images. In terms of computational complexity SFR outperforms both spin and tensor images for use as a rejector.

\subsection{Spherical Face Representation and Rejection classifier (SFR)}

Spherical face Representation and rejection classifier are dependent factors. A rejection classifier is structured in such a way with SFR, that it quickly excludes a large proportion of the candidate classes with higher probability rate and quickly eliminates a large number of candidate faces at an early stage for efficient recognition of larger galleries. The effectiveness of rejection classifier is measured by following equation.

$$
\operatorname{Perf}(\mu)=\frac{P_{a \in S}(\mu(a))}{G}
$$
..(eqn 1)

Where ${ }_{a} \in \mathrm{s}$ returns a set of class labels and $\mathrm{G}$ is the size of gallery. When compared to brute force matching approach our rejection classifier approach would reduce the comparison to $0.03 \%$ which proves to be a better approach. Remaining faces were verified using region-based matching approach were robust to facial expressions. As the segmented eyes, forehead and nose regions were relatively less sensitive to expressions and matches were performed separately using a novel Iterative Closest Point (ICP) algorithm. The results of all the matching engines are fused at the metric level to achieve higher accuracy. Performance evaluation is done on FRGC v2.0 benchmark data. Multimodal hybrid algorithm performed better than others by achieving 99.74 percent and 98.31 percent verification rates at a 0.001 false acceptance rate (FAR) and Identification rates of 99.02 percent and 95.37 percent for probes with a neutral and a non neutral expression

Facial recognition system identifies a person based on the feature extraction of facial components with the help of known faces. In this work, we perform face recognition system based on known faces with the help of matching algorithm. One of the methods is to perform the comparison process with the available facial dataset. Our existing work presented a multimodal (2D and 3D) face recognition algorithm and Hybrid matching algorithm based on both feature based and holistic metrics. 


\subsection{Feature based Multimodal Face Recognition System Architecture}

The architectural diagram of our proposed work is as presented in fig2. The Feature based Multimodal Face Recognition system consists of four phases. The first phase consists of face detection.

The face is detected using feature invariant methods and template matching methods. It tries to find invariant features of a face that includes removal of unwanted pixels from the images based on the geometric value. Template matching process compares input images with stored features.

The second phase consists of feature extraction extracts relevant feature from the data which is performed using PCA technique and features like shape of the eyes, nose and jaw are selected by discarding the non relevant feature using branch and bound method that causes smallest classification error by way of minimizing the dimensionality and complexity in execution time.

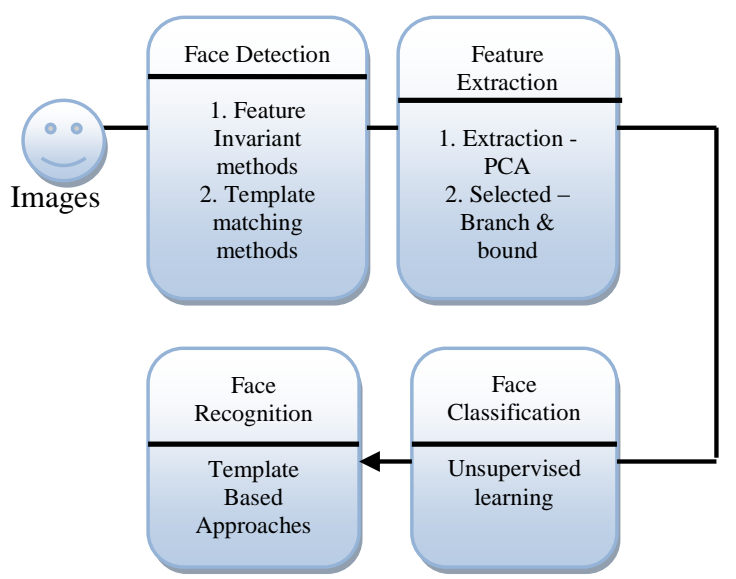

Fig 2. Architecture of Feature based Multimodal Face Recognition System

The third phase consists of Face Classification that classifies the face based on the unsupervised learning by using novel kmeans clustering. The last phase involves the face recognition approach that is performed using template based approaches where the input images are compared with a set of templates stored in databases as features.

\subsection{Feature Extracted Multimodal Face Recognition System - Algorithm}

Step 1: Scan the input image for face detection.

Step 2: Perform Face Detection using feature invariant methods by discarding angle and position.

Step 2.1: Perform template matching methods which compare input images with stored patterns of features.

Step 3: Perform Feature Extraction based on the extraction process using PCA.

Step 3.1: Features like shape of the eyes, nose and jaw are selected using branch-and-bound technique by minimizing the dimensionality and complexity.

Step 4: Face classification is performed using novel k-mean cluster of unsupervised learning approach.

Step 5: Template matching process is performed for the features using template based approaches.

\section{EXPERIMENTAL RESULTS AND DISCUSSION}

The experimental evaluation of Efficient Multimodal 2D-3D Hybrid Approach to Automatic Face Recognition are conducted and presented herewith. The performance of SFR is compared to the spin images when used as rejection classifiers. The probes were performed with both neutral and non-neutral expression. For probes with a neutral expression, the spin images performed better whereas for probes with non-neutral expression the SFR performed better.

The results performed based on the classifier shows that SFRbased classifier proves to be more efficient than the spin image classifier. A Matlab implementation on a $2.3-\mathrm{GHz}$ Pentium IV machine took $6.2 \mathrm{~ms}$ to construct an SFR of a probe, match it with the 455 SFRs in the gallery, and reject a subset of the gallery, whereas the spin images took $2,363 \mathrm{~ms}$ for the same purpose.

Our work uses False Acceptance Rate (FAR) and False Rejection Rate (FRR) are the two metrics used for measuring the performance of feature based multimodal face recognition system. The results of FAR and FRR are obtained from equation 2 and equation 3 respectively. Fig 3 shows the performance results of the False Acceptance Rate measured for both the works MFRS and FBMFRS

$$
\text { FAR }=\frac{\text { Number of images accepted } * 100}{\text { Number of images tested }}
$$

2)

$\mathrm{FRR}=\frac{\text { No. of original images rejected } * 100}{\text { No. of original images tested }}$ 3)

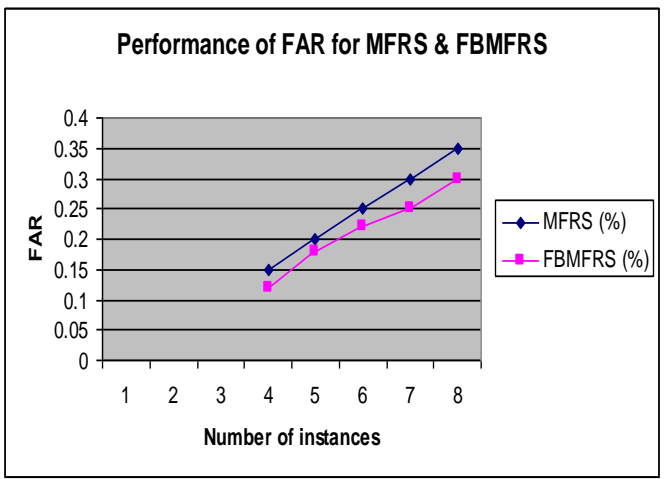

Fig 3. Performance of FAR for multimodal face recognition and feature based multimodal face recognition systems.

From fig 3, it is noted that as the number of instances increases, the FAR for MFRS gets increased whereas the FAR for FBMFRS outperforms when compared to the existing work. The FAR rate for FBMFRS is lesser when compared to MFRS by reducing complexity. 


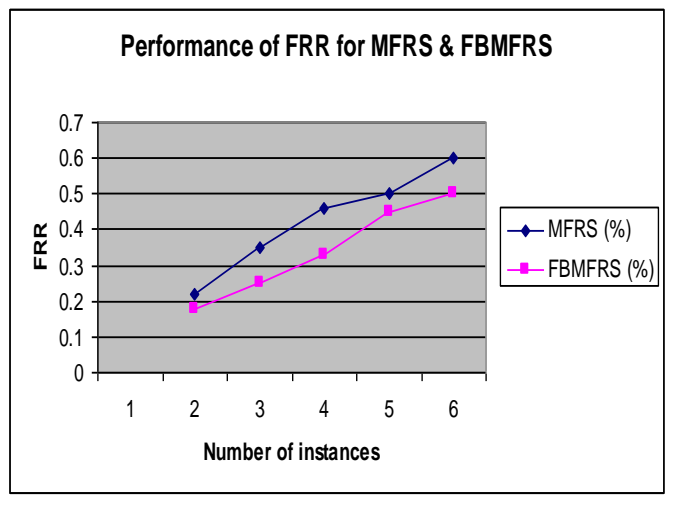

Fig 4. Performance of FRR for multimodal face recognition and feature based multimodal face recognition systems.

In fig 4, $\mathrm{X}$ axis represents number of training templates and $\mathrm{Y}$ axis represents the False Rejection Rate for Multimodal Face Recognition System and Feature Based Multimodal Face Recognition System. An increase in number of training templates shows a gradual increase in the FRR value. The experimental results implements that the proposed algorithm FBMFRS is much better than the MFRS.

\section{CONCLUSION}

Here a method Feature Based Multimodal Face Recognition System is presented and demonstrated the performance of False Acceptance Rate and False Rejection Rate on various benchmark data sets of facial recognition models using known faces with features such as shape of the eyes, nose and jaw. Our contributions inclusion of feature invariant methods that discards angle and position and selection of features using branch and bound technique.

Several experiments have been conducted with image size variant to $256 * 256$ pixels of image in order to evaluate the performance of the algorithm. The FAR is improved for Feature Based Multimodal Face Recognition System with reduction of $1.05 \%$ and FRR is improved with reduction of $1.25 \%$ times as compared to MFRS. This in turn reduces the complexity.

\section{REFERENCES}

[1]. D. Lowe, 2004, "Distinctive Image Features from ScaleInvariant Key Points," Int'l J. Computer Vision, vol. 60, no. 2, pp. 91-110.

[2]. P. Yan and K. Bowyer, 2004, "Empirical Evaluation of Advanced Ear Biometrics," Proc. IEEE Computer Vision and Pattern Recognition Workshops, pp. 308-313.

[3]. T. Maurer, D. Guigonis, I. Maslov, B. Pesenti, A. Tsaregorodtsev, D. West, and G. Medioni, 2005, "Performance of Geometrix ActiveID 3D Face Recognition Engine on the FRGC Data," Proc. IEEE Workshop Face Recognition Grand Challenge Experiments.

[4]. G. Passalis, I. Kakadiaris, T. Theoharis, G. Tederici, and N. Murtaza, 2005, "Evaluation of 3D Face Recognition in the Presence of Facial Expressions: An Annotated Deformable Model Approach," Proc. IEEE Workshop Face Recognition Grand Challenge Experiments.

[5]. A.S. Mian, M. Bennamoun, and R.A. Owens, 2006, “A Novel Representation and Feature Matching Algorithm for Automatic Pairwise Registration of Range Images," Int'1 J. Computer Vision, vol. 66, pp. 19-40.

[6]. D. Lin and X. Tang, 2006, "Recognize High Resolution Faces: From Macrocosm to Microcosm," Proc. IEEE Computer Vision and Pattern Recognition, pp. 13551362.

[7]. A.S. Mian, M. Bennamoun, and R.A. Owens, 2006, "2D and 3D Multimodal Hybrid Face Recognition," Proc. European Conf. Computer Vision, part III, pp. 344-355.

[8]. X. Lu, A.K. Jain, and D. Colbry, 2006, "Matching 2.5D Scans to 3D Models," IEEE Trans. Pattern Analysis and Machine Intelligence, vol. 28, no. 1, pp. 31-43, Jan.

[9]. A.S. Mian, M. Bennamoun, and R.A. Owens, 2006 , "Automatic 3D Face Detection, Normalization and Recognition," Proc. Third Int'l Symp. 3D Data Processing, Visualization and Transmission.

[10]. A.S. Mian, M. Bennamoun, and R.A. Owens, Oct 2006 , "Three-Dimensional Model-Based Object Recognition and Segmentation in Cluttered Scenes," IEEE Trans. Pattern Analysis and Machine Intelligence, vol. 28, no. 10, pp. 1584-1601

[11]. P.J. Phillips, P.J. Flynn, T. Scruggs, K.W. Bowyer, and W. Worek, 2006, "Preliminary Face Recognition Grand Challenge Results," Proc. Int'l Conf. Automatic Face and Gesture Recognition. 